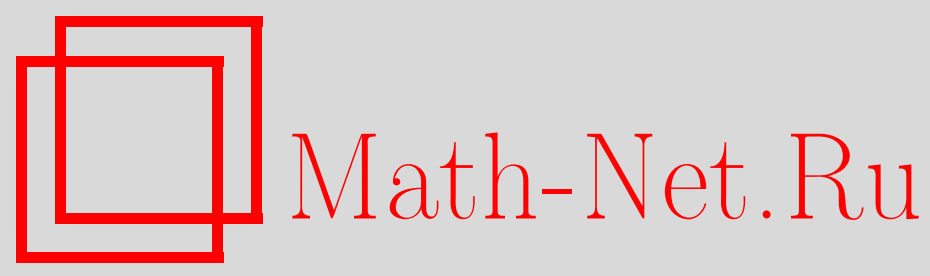

В. И. Данченко, Д. Я. Данченко, О приближении наипростейшими дробями, Матем. заметки, 2001, том 70, выпуск 4, 553-559

DOI: https://doi.org/10.4213/mzm767

Использование Общероссийского математического портала Math-Net.Ru подразумевает, что вы прочитали и согласны с пользовательским соглашением http://www . mathnet.ru/rus/agreement

Параметры загрузки:

IP : 52.87 .193 .239

26 апреля 2023 г., 10:33:55

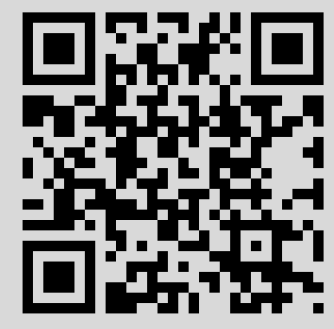




\section{О ПРИБЛИЖЕНИИ НАИПРОСТЕЙШИМИ ДРОБЯМИ}

\section{В. И. Данченко, Д. Я. Данченко}

В заметке рассматриваются некоторые задачи о равномерной аппроксимации на компактах комплексной плоскости комплекснозначных непрерывных функций $f(z)$ наипростейшими дробями вида $\Theta_{n}(z)=\sum_{j=1}^{n} 1 /\left(z-z_{j}\right)$. Показано, например, что наилучшие приближения функции $f$ дробями $\Theta_{n}$ имеют тот же порядок малости, что и наилучшие приближения многочленами степени $\leqslant n$.

Библиограбфия: 7 названий.

1. В заметке рассматриваются некоторые задачи аппроксимации на множествах $K$ расширенной комплексной плоскости $\overline{\mathbb{C}}$ комплекснозначных функций $f$ наипростейшими дробями (этот термин предложен Долженко) вида

$$
\Theta_{n}(z)=\sum_{j=1}^{n} \frac{1}{z-z_{j}}=\frac{Q_{n}^{\prime}(z)}{Q_{n}(z)}
$$

где $Q_{n}(z)$ - некоторый многочлен степени $n$.

Пусть $K$-произвольноемножество на $\overline{\mathbb{C}}$. Для определенной на $K$ функции $f$ положим

$$
\|f\|_{K}=\sup _{z \in K}|f(z)|
$$

Через $\rho\left(\Theta_{n}, K\right)$ будем обозначать евклидово расстояние между множеством $\left\{z_{j}\right\}$ полюсов функции $\Theta_{n}$ и $K$. При $n=1,2, \ldots, 0<r \leqslant \infty$ для функции $f \in C(K)$ определим наилучшее приближение

$$
\Psi_{n}(f ; K ; r)=\inf \left\{\left\|f-\Theta_{n}\right\|_{K}: \operatorname{deg} \Theta_{n} \leqslant n, \rho\left(\Theta_{n}, K\right) \leqslant r\right\}
$$

Указанная аппроксимация в ряде случаев тесно связана с хорошо известной проблемой Горина (см. [1], [2]) о скорости убывания наименьших расстояний

$$
\rho_{n}(K)=\inf \left\{\rho\left(\Theta_{n}, K\right):\left\|\Theta_{n}\right\|_{K} \leqslant 1\right\}, \quad n \rightarrow \infty .
$$

Работа выполнена при поддержке Российского фонда фундаменталшных исследований, гранты № 99-01-00119 и № 00-01-00342. 
Так, для случая прямой $K=\mathbb{R}^{1}$ в [3] показано, что $\rho_{n}\left(\mathbb{R}^{1}\right)=\Psi_{n}\left(0 ; \mathbb{R}^{1} ; 1\right) \asymp \ln \ln n / \ln n$ (первое равенство, очевидно, следует из того, что при фиксированном $\lambda \in \mathbb{C}$ функция $\lambda \Theta_{n}(\lambda z)$ также является наипростейшей дробью от $\left.z\right)$. Другие результаты по исследованию величин $\rho_{n}(K), \Psi_{n}(f ; K ; r)(0<r \leqslant \infty)$ для различных множеств $K \subset \overline{\mathbb{C}}$ см. в работах Горина, Николаева, Гельфонда, Кацнельсона, В. И. Данченко и Д. Я. Данченко, Косухина (см., например, [1]-[7]).

В общей ситуации задачу апшроксимации на $K$ функций $f \in C(K)$ можно ставить как в случае дробей $\Theta_{n}$ со свободными полюсами, так и в случае каких-либо ограничений на их расположение. В первом случае дополнительно возникает задача о связи величин $\rho\left(\Theta_{n}, K\right)$ и $\left\|f-\Theta_{n}\right\|_{K}$. В [3], [6] показано, например, что если $K=\partial \Delta-$ единичная окружность, то из $\Psi_{n}(0 ; \partial \Delta ; r) \leqslant M$ вытекает, что $r \geqslant \ln n /(3 n)$ при $M \geqslant 1$ и $r \geqslant \mathrm{const} \cdot M^{-1 / n}$ при $M \leqslant 1\left(n \geqslant n_{0}\right)$. Отметим, что аппроксимацию дробями $\Theta_{n}(z)$ можно использовать в теории потенциала при распределении зарядов, имеющих на $K$ заданный суммарный комплексньй потенциал $f$, в приближенных вычислениях (см. неравенства $(3),(4))$ и т.д. Кроме того, оценки расстояний $\rho_{n}(K)$ играют немаловажную роль в вопросах устойчивости динамических систем.

2. Скажем, что некоторое множество $G \subset \mathbb{C}$ принадлежит классу $\Omega_{r}, r>0$, если оно содержит точку $z_{0}=0$ и любую точку $z \in G$ можно соединить с $z_{0}$ лежащей на $G$ спрямляемой кривой длины $\leqslant r$. Однозначную аналитическую на множестве $G \in \Omega_{r}$ (т.е. в некоторой окрестности множества $G$ ) функцию $f$ будем называть допустимой на $G$, если $\|f\|_{G} \leqslant 1$ и интеграл

$$
\alpha(f ; z):=\int_{0}^{z} f(t) d t
$$

взятый по всем кривым, лежащим в $G$ и имеющим концевые точки 0 и $z$, определяет также однозначную аналитическую функцию от $z$. Положим

$$
q_{1, n}(f ; z)=\sum_{j=0}^{n} \frac{\alpha^{j}(f ; z)}{j !}, \quad q_{2, n}(f ; z)=\sum_{j=0}^{n}(-1)^{j} \frac{\alpha^{j}(f ; z)}{j !} .
$$

Лемма 1. Пусть $r>0 u G \in \Omega_{r}$. Пусть $f(z)$ - отличная от нуля допустимая на $G$ функиия. Тогда при $s=1,2$ и $\theta_{s, n}(f ; z):=q_{s, n}^{\prime}(f ; z) / q_{s, n}(f ; z) n p u n \geqslant 5 r$ имеем

$$
\left\|f+(-1)^{s} \theta_{s, n}(f ; z)\right\|_{G} \leqslant \frac{2 e^{r} r^{n}}{n !} .
$$

ДокаЗАтЕльство. Рассмотрим случай $s=1$. Очевидно, $|\alpha(f ; z)| \leqslant r$ на $G$. Далее, для функции $q_{1, n}(f ; z)$ находим

$$
\delta(z)=f(z)-\theta_{1, n}(f ; z)=\frac{1}{n !} \frac{f(z)}{q_{1, n}(f ; z)} \alpha^{n}(f ; z) .
$$

Отсюда

$$
\begin{aligned}
\left|q_{1, n}(f ; z)\right| & \geqslant e^{\operatorname{Re} \alpha(f ; z)}-\left|\sum_{j=n+1}^{\infty} \frac{\alpha^{j}(f ; z)}{j !}\right| \geqslant e^{-r}-\frac{r^{n+1}}{(n+1) !}\left(1+\frac{r}{n+2} e^{r}\right) \\
& \geqslant e^{-r}-\frac{1}{\sqrt{2 \pi n}} \frac{n+2+r}{n+2}\left(\frac{r e}{n+1}\right)^{n+1} e^{r} \geqslant \frac{1}{2} e^{-r}
\end{aligned}
$$


при $z \in G$ и $n \geqslant 5 r$. Отсюда получается нужная оценка сверху для $|\delta(z)|$ на $G$. При $s=2$ доказательство аналогично.

В [6] авторами доказана

ТЕОрема 1. Пусть $r>0 u G \in \Omega_{r}$. Далее, пусть $P(z)$ - отличный от тохдественного нуля многочлен степени $N \geqslant 0 u\|P\|_{G} \leqslant 1$. Тогда при любых натуральных $n \geqslant 5 r$ имеем

$$
\Psi_{(N+1) n}(P ; G ; \infty) \leqslant e^{r}\left(\frac{e r}{n}\right)^{n} .
$$

Точнее, при $s=1,2$ существует наипростейщая дробь $\Theta(z)=Q^{\prime}(z) / Q(z)$ cmепени $(N+1) n$ такая, что

$$
\left\|P+(-1)^{s} \Theta\right\|_{G} \leqslant \sqrt{\frac{2}{\pi}} e^{r} n^{-1 / 2}\left(\frac{e r}{n}\right)^{n} .
$$

Теорема 1 есть непосредственное следствие леммы 1 при $f=P$ и $q_{s}(f ; z)=Q(z)$. Аналогично из леммы 1 получается

Теорема 2. Пусть $r>0, G \in \Omega_{r}, R(z)$ - отличная от тождественного нуля рачиональная функиия степени $N \geqslant 0,\|R\|_{G} \leqslant 1$. Пусть, кроме того, $R$ имеет однозначный интеграл на $\mathbb{C}(u$, в частности, является допустимой функиией). Тогда при любых натуральных $n \geqslant 5 r$ существуют наипростейшие дроби $\Theta_{1}(z)$ $u \Theta_{2}(z)$ степеней $\leqslant(N+1) n$ такие, что

$$
\left\|R+\Theta_{1}-\Theta_{2}\right\|_{G} \leqslant \frac{2 e^{r} r^{n}}{n !} .
$$

На основании теоремы Мергеляна и теоремы 1 получается

Теорема 3. Пусть $K$ - компакт, имеющий связное дополнение на $\overline{\mathbb{C}}$. Тогда любая функиия $f$, непрерывная на $K$ и аналитическая во внутренних точках $K$, может быть равномерно приближсена слюбой точностью на $K$ наипростейшими дробями вида $\Theta=Q^{\prime} / Q$, где $Q$ - многочлен. Кроме того, если $K \in \Omega_{r}(r>0)$, $E_{n}=E_{n}(f, K)$ - наилучшее равномерное приближение на $K$ функиии $f \in C(K)$ множеством многочленов степени $\leqslant n u 0<E_{n} \rightarrow 0(n \rightarrow \infty)$, то

$$
\Psi_{n \ln \left(1 / E_{n}\right)}(f ; K ; \infty)<2 E_{n}, \quad n \geqslant n_{0}(r) .
$$

В частности, если $f$ аналитична на континууме $K$ со связным дополнением, то по теореме Бернштейна-Уолша имеем $E_{n}<\lambda^{n}\left(n \geqslant n_{1}(\lambda, f)\right)$ с некоторым $\lambda=\lambda(f) \in(0,1)$ и из (4) находим

$$
\Psi_{n}(f ; K ; \infty)<C \lambda_{1}^{\sqrt{n}}, \quad \lambda_{1}=\lambda^{1 / \sqrt{-\ln \lambda}}, \quad n \geqslant n_{2}(r, \lambda, f) .
$$

Другие обратные теоремы, основанные на идеях работы [6], см. в работе Косухина [7].

Возникает задача о возможности аналогичной апроксимации специальными дробями вида

$$
\Theta\left(V,\left\{z_{j}\right\} ; z\right)=\sum_{j=1}^{n}\left(z-z_{j}\right)^{-1} V\left(z_{j}\right),
$$


где вес $V$ зависит лишь от $z_{j}$. K примеру, если $V(z)=1 / z$, то теорема 3 остается справедливой и для дробей $\Theta\left(V,\left\{z_{j}\right\} ; z\right)$, поскольку в этом случае $z \Theta\left(V,\left\{z_{j}\right\} ; z\right)=\Theta(0)+$ $\Theta(z)$, где $\Theta=Q^{\prime} / Q, Q(z)=\prod_{j=1}^{n}\left(z-z_{j}\right)$. Если же $V(z)=z$, то апшроксимация невозможна даже в простейшем случае $f(z)=i$ и $K=\{z:|z| \leqslant 1\}$ (поскольку на окружности $\partial K$ всегда найдется точка $z_{0}$, в которой значение $\Theta\left(V,\left\{z_{j}\right\} ; z_{0}\right)$ вещественно; например, оно вещественно в каждой точке $z_{0}$ максимума модуля многочлена $\left.Q(z)\right)$.

3. Как видно из $(2)$, если $P(0) \neq 0$, то дробь $\Theta(z), \operatorname{deg} \Theta \leqslant(N+1) n$, интерполирует многочлен $P$ в точке $z=0$ с кратностью $n$. Можно построить наипростейшую дробь $\Theta$ (дробь Паде), интерполирующую $P$ в нуле с той же кратностью $n$, но при этом имеющую степень $\operatorname{deg} \Theta=n$. Точнее, имеет место следующее

УТВЕРЖДЕНИЕ. Для произвольного набора чисел $\left\{a_{\nu}\right\}, \nu=0,1, \ldots$, при каждом натуральном $n$ существует дробь $\Theta_{n}=Q_{n}^{\prime} / Q_{n}$ степени $n$ такая, что

$$
\Theta_{n}^{(\nu)}(0)=a_{\nu}, \quad \nu=\overline{0, n-1}
$$

Кроме того, соответствуюшие многочлены $Q_{n}$ могут быть записаны в виде

$$
Q_{1}(z)=1+a_{0} x, \quad \ldots, \quad Q_{n+1}(z)=Q_{n}(z)+\beta_{n+1} z^{n+1}
$$

с некоторыми константами $\beta_{n+1}$.

Действительно, пусть среди чисел $\left\{a_{1}, \ldots, a_{n-1}\right\}$ есть отличные от нуля и $z_{1}, \ldots, z_{n}-$ корни искомого многочлена $Q_{n}(z)=1+\beta_{1} z+\cdots+\beta_{n} z^{n}$. Из (5) найдем моменты (степенные суммы)

$$
S_{\nu}=S_{\nu}\left(Q_{n}\right)=\sum_{j=1}^{n} z_{j}^{-\nu}=-\frac{\Theta_{n}^{(\nu-1)}(0)}{\nu !}=-\frac{a_{\nu-1}}{\nu !}, \quad \nu=\overline{1, n}
$$

Коэффициенты $\beta_{\mu}$ многочлена $P(z)=z^{n} Q_{n}(1 / z)=z^{n}+\beta_{1} z^{n-1}+\cdots+\beta_{n}$ совпадают с точностью до знака с элементарными симметрическими многочленами от переменных $z_{j}^{-1}$ и выражаются рекуррентно через указанные моменты по формуле Ньютона

$$
\beta_{1}=-S_{1}, \quad \beta_{\mu}=-\mu^{-1}\left(S_{\mu}+\sum_{j=1}^{\mu-1} S_{\mu-j} \beta_{j}\right), \quad \mu=\overline{2, n}
$$

Итак, $Q(z)=z^{n} P(1 / z)$. Из построения видно, что все коэффициенты многочленов $Q_{n}$ и $Q_{n+1}$ с одинаковыми номерами совпадают.

4. Пусть моменты $S_{\nu}$ вещественны и $S_{\nu} \leqslant 1$ при $\nu=\overline{1, n}$. Покажем, что все корни $\left\{z_{j}\right\}$ многочлена $Q$ лежат вне прямолинейного отрезка $[0,1]$. Легко видеть, что коэффициенты многочлена $Q$ являются однородными функциями от переменных $S_{1}, \ldots, S_{n}$; точнее, $\beta_{\mu}=\beta_{\mu}\left(S_{1}, \ldots, S_{n}\right)=\sum A(k, p ; \ldots ; m, q) S_{k}^{p} \cdots S_{m}^{q}$ с некоторыми действительными коэффициентами $A(k, p ; \ldots ; m, q)$, где суммирование распространяется на все те индексы и степени, для которых $k p+\cdots+m q=\mu$. Кроме того, если $S_{k} \leqslant 0$ при всех 
$k=\overline{1, n}$, то по индукции из (6) получаются неравенства $\beta_{\mu} \geqslant 0$ при всех $\mu=\overline{1, n}$. При $\mu=\overline{1, n}$ положим

$$
\beta_{\mu}^{(1)}=\beta_{\mu}\left(S_{1}-1, \ldots, S_{n}-1\right)=\sum A(k, p ; \ldots ; m, q)\left(S_{l}-1\right)^{p} \cdots\left(S_{m}-1\right)^{q} .
$$

Легко проверяется (по индукции), что из (6) вытекают рекуррентные соотношения $\beta_{\mu}^{(1)}$ $\beta_{\mu-1}^{(1)}=\beta_{\mu}$, где $\mu=\overline{1, n}$ и $\beta_{0}^{(1)}=1$. Отсюда с учетом предположения в начале п. 4 имеем

$$
Q(z)=(1-z) \sum_{\mu=0}^{n-1} \beta_{\mu}^{(1)} z^{\mu}+\beta_{n}^{(1)} z^{n}
$$

где все коэффициенты $\beta_{\mu}^{(1)}$ неотрицательны. Итак, на $[0,1]$ нет корней многочлена $Q$.

Лемма 2. Пусть $Q_{n}(z)=1+\beta_{1} z+\cdots+\beta_{n} z^{n}-$ многочлен с корнями $z_{1}, \ldots, z_{n}$ и модули всех моментов

$$
S_{\nu}=\sum_{j=1}^{n} z_{j}^{-\nu}, \quad \nu=\overline{1, n}
$$

не превосходят 1/2. Тогда все корни $\left\{z_{j}\right\}$ лежсат вне единичного круга.

ДокАЗАтЕльство. Действительно, из существования корней многочлена $Q(z)$ на некотором фиксированном радиальном отрезке $\rho e^{i \varphi}, 0 \leqslant \rho \leqslant 1$, следовало бы существование корней многочлена $q(\zeta)=Q\left(\zeta e^{i \varphi}\right) \bar{Q}\left(\zeta e^{i \varphi}\right)$ на отрезке $[0,1]$. Но многочлен $q$, очевидно, удовлетворяет предположениям начала п. 4 и не имеет корней на $[0,1]$.

5. Пусть

$$
f(z)=\sum_{\mu=1}^{\infty} \frac{a_{\mu} z^{\mu}}{\mu !}
$$

- регулярная в окрестности точки $z=0$ функция. Тогда из (6) и из формул Ньютона

$$
S_{\mu}+\sum_{j=1}^{n} S_{\mu-j} \beta_{j}=0, \quad \mu>n
$$

получаем соотношения, определяющие последовательность многочленов

$$
Q_{n}=1+\sum_{\mu=1}^{n} \beta_{\mu} z^{\mu}
$$

Паде (см. (5)) для функции $f$, а также моменты $S_{\mu}=S_{\mu}\left(Q_{n}\right)$ с номерами $\mu>n$ :

$$
\left(1+\sum_{\mu=1}^{n} \beta_{\mu} z^{\mu}\right)\left(1-\sum_{\mu=1}^{n} \frac{a_{\mu}}{\mu !} z^{\mu}+\sum_{\mu=n+1}^{\infty} S_{\mu} z^{\mu}\right)=1-\beta_{2} z^{2}-\cdots-(n-1) \beta_{n} z^{n}
$$


Отсюда находим

$$
Q_{2}(z)=Q_{1}(z)+\frac{1}{2}\left(a_{1}+a_{0}^{2}\right) z^{2}, \quad Q_{3}(z)=Q_{2}(z)+\frac{1}{6}\left(2 a_{2}+3 a_{0} a_{1}+a_{0}^{3}\right) z^{3}
$$

И т.д.

В случае простых различных узлов интерполяции $v_{\nu}, \nu=\overline{1, n}$, для определения многочлена $Q(z)=z^{n}+\alpha_{n-1} z^{n-1}+\cdots+\alpha_{0}$ условия интерполяции $\Theta\left(v_{\nu}\right)=a_{\nu}$ записываются в виде системы линейных уравнений относительно неизвестных $\alpha_{j}$ :

$$
\sum_{j=0}^{n-1}\left(j-a_{\nu} v_{\nu}\right) v_{\nu}^{j-1} \alpha_{j}=v_{\nu}^{n-1}\left(-n+a_{\nu} v_{\nu}\right), \quad \nu=\overline{0, n-1}
$$

На практике при интерполяции некоторых аналитических функций $f$ вопрос о разрешимости системы легко решается, если интерполяцию производить на линии уровня $\left|v_{\nu} f\left(v_{\nu}\right)\right|=$ const.

6. Рассмотрим вопрос об апшроксимации регулярной в единичном круге $\Delta=\{z$ : $|z|<1\}$ функции

$$
f(z)=\sum_{\nu=0}^{\infty} \alpha_{\nu}(f) z^{\nu}
$$

с условием $\left|\alpha_{\nu}(f)\right| \leqslant M<\infty$ ее наипростейшими дробями Паде $\Theta_{n}(z)=Q_{n}^{\prime}(z) / Q_{n}(z)$, построенными в п. 3. Отметим, к примеру, что указанному ограничению удовлетворяют функции класса Харди $H^{1}(\Delta)$ с нормировкой $\|f\|_{H^{1}(\Delta)} \leqslant M$.

Рассмотрим функцию $F(z)=f(z) /(2 M)$. Запишем условия интерполящии: $\nu ! S_{\nu+1}=$ $-\Theta_{n}^{(\nu)}(0)=-\nu ! \alpha_{\nu}(F), \nu=\overline{0, n-1}$. Отсюда $\left|S_{\nu}\right| \leqslant 1 / 2$ и по лемме 2 все корни многочлена $Q_{n}(z)$ лежат вне $\Delta$. Итак, при $|z|<1$ имеем

$$
\left|F(z)-\Theta_{n}(z)\right| \leqslant \sum_{\nu=n}^{\infty}\left(n+\frac{1}{2}\right)|z|^{\nu}
$$

Таким образом, доказана следующая

Теорема 4. Пусть $f(z)=\sum_{\nu=0}^{\infty} \alpha_{\nu}(f) z^{\nu}$ - регулярная в $\Delta$ функиия, причем $\left|\alpha_{\nu}(f)\right| \leqslant M(\nu \in \mathbb{N})$. Тогда наипростейшие дроби Паде $\Theta_{n}$ функиии $F(z)=f(z) /(2 M)$ равномерно сходятся $\kappa F$ на любом круге $\Delta_{\lambda}=\{z:|z| \leqslant \lambda<1\}$ и при этом

$$
\left\|F-\Theta_{n}\right\|_{\Delta_{\lambda}} \leqslant \frac{1+n}{1-\lambda} \lambda^{n}
$$

Следующий пример показьвает, что значительное усиление теоремы 4 невозможно. Для функции $f(z)=\sum_{j=0}^{\infty} z^{j}=1 /(1-z)$ дроби $\theta_{n}=q_{n}^{\prime} / q_{n}$ с $q_{n}(z)=\sum_{j=0}^{n} z^{j}$ являются дробями Паде и при $|z|^{<}<1$ имеем $\left|\theta_{n}(z)-f(z)\right|=(n+1)|z|^{n} /\left|1-z^{n+1}\right|$.

7. В заключение докажем одно частное утверждение об оценке снизу величины расстояний $\rho\left(\Theta_{m}, K\right)$ в зависимости от $\left\|f-\Theta_{m}\right\|_{K}$. 
Теорема 5. Пусть $P$ - многочлен степени $n \geqslant 1, Q_{n k}$ - многочлен степени $n k \geqslant 1$ и при некоторых $\varepsilon \in(0,1)$ и $а \in\left(0, k^{\varepsilon}\right)$ на единичном круге $\Delta$ выполнена оценка

$$
\left\|P-\Theta_{n k}\right\|_{\Delta} \leqslant a^{k} k^{-k} .
$$

Тогда при достаточно больших $k$ имеем $\rho\left(\Theta_{n k}, \partial \Delta\right) \geqslant C \cdot k$, где $C$ зависит лишь om $a, \varepsilon,\|P\|$.

ДокАЗАТЕЛЬСтво. Действительно, из (7) и из теоремы 1 следует, что существует некоторый многочлен $q=T_{n k} Q_{n k}$ степени $2 n k$, модуль логарифмической проиводной которого на $\partial \Delta$ ограничен величиной $A_{1}\left(e^{k}+a^{k}\right) / k^{k}$. Остается использовать результат из [6] (см. п. 1): $\rho\left(q^{\prime} / q, \partial \Delta\right) \geqslant$ const $\cdot M^{-1 / n}$.

\section{СПИСОК ЦИТИРОВАННОЙ ЛИТЕРАТУРЫ}

[1] Горин Е.А. Частично гипоэллиптические дифференциальные уравнения в частных производных с постоянными коэффициентами // Сиб. матем. ж. 1962. № 4. С. 506-508.

[2] Гельфонд А. О. Об оценке мнимых частей корней многочленов с ограниченными производньми от логарифмов на действительной оси // Матем. сб. 1966. Т. 71(113). С. 289-296.

[3] Данченко В.И. Оценки расстояний от полюсов логарифмических производных многочленов до прямых и окружностей // Матем. сб. 1994. Т. 185. № 8. С. 63-80.

[4] Кацнельсон В. Э. О некоторых операторах, действующих в пространствах, порожденных функциями $\frac{1}{z-z_{k}} / /$ Теория функций, функцион. анализ и их прилож. 1967. № 4. С. 58-66.

[5] Николаев Е. Г. Геометрическое свойство корней многочленов // Вестн. МГУ. Сер. Матем., мех. 1965. № 5. С. 23-26.

[6] Данченко В.И., Данченко Д.Я. О равномерном приближении логарифмическими производными многочленов // Теория функций, ее приложения и смежные вопросы. Тезисы докл. шкколы-конференции: Изд-во Казанского университета, 1999. С. 74-77.

[7] Косухин О.Н. Аналог неравенства Джексона для аппроксимаций наипростейшими дробями // Современные проблемы теории функций и их приложения. Тр. 10-й Саратовской зимней шшколы: Изд-во Саратовского университета, 2000. С. 68-69. 